\title{
The effects of group composition and evaluation on task performance
}

\author{
JOHN J. SETA, PAUL B. PAULUS, and HAL T. RISNER \\ University of Texas, Arlington, Texas 76019
}

\begin{abstract}
The effects of the presence of coactors working on the same and different tasks were examined. Subjects were run under either high- or low-evaluative conditions and in either a mixed group (half worked on a maze task while half worked on a multiplication task) or in a homogeneous group (all subjects worked on the maze task). A significant Evaluation by Group Composition interaction was obtained. Under high-evaluative conditions subjects in the mixed condition performed significantly better on the maze task than did subjects on the homogeneous condition, while under low-evaluative conditions, the mixed and homogeneous subjects did not differ in their maze performance. The results were discussed in terms of learned drive theory and incentive motivation.
\end{abstract}

Past research has demonstrated that an individual's task performance can be affected by the presence of others (see Cottrell, 1972, for a review). This research has employed two paradigms, audience and coaction. In the audience paradigm, individuals perform a task in the presence of observers, while in the coaction paradigm a group of individuals concurrently and individually perform an identical task. Zajonc (1965) has argued that the mere presence of others increases an individual's general level of arousal, which in turn increases the tendency to emit dominant responses. If the dominant responses are correct (as with well-learned tasks), heightened arousal will result in improved performance by an individual; if the dominant responses are incorrect (as with tasks that are not well learned), arousal will result in impaired performance. Cottrell (1972) has modified Zajonc's (1965) conceptualization by arguing that mere presence is not a sufficient condition for increased arousal. He proposes that the mere presence of others has nondirective energizing effects only if the presence of others creates anticipations of positive or negative outcomes.

Seta, Paulus, and Schkade (1976) have extended this position by proposing that others can increase, decrease, or have no effect on arousal depending on whether they are positive, negative, or neutral stimuli. That is, others can be a source of arousal reduction when their presence decreases the intensity or the probability of an aversive outcome and/or increases the probability or intensity of a positive outcome. Others can also be a source of

This research was supported in part by a grant from the Organized Research Fund of the University of Texas at Arlington. John J. Seta is now at the Department of Psychology, University of North Carolina at Greensboro. Requests for reprints should be sent to Paul B. Paulus, Department of Psychology, University of Texas at Arlington, Arlington, Texas 76019. The assistance of Angela Annis in this research is gratefully acknowledged. In addition, special thanks is given to B. B. Bruno for his conceptual inspiration. arousal induction when their presence increases the probability or intensity of an aversive outcome and/or decreases the probability or intensity of a positive outcome. In support of this position, research employing an audience or coaction paradigm has indicated that others can decrease arousal (Seta et al., 1976), increase arousal (e.g., Hunt \& Hillery, 1973; Zajonc \& Sales, 1966), or have no effect on arousal (e.g., Paulus \& Murdoch, 1971).

Coaction studies have typically used situations in which subjects work concurrently and independently on the same task. However, in many nonlaboratory situations, individuals do not work on the same task but work concurrently and independently on different tasks. To our knowledge, research thus far has not investigated this situation even though in these situations the presence of others could also be a potential source of arousal induction or reduction. The present experiment was designed to investigate an individual's performance on a complex task in groups of different compositions. Subjects worked either in a mixed group (half the individuals in the group worked on a maze task while the other half worked on a multiplication task) or in a homogeneous group (all individuals in the group worked on a maze task). In addition, half of the subjects were run under conditions stressing evaluative comparison, while the other half were run under lowevaluative conditions. The primary dependent measure was the number of errors each subject committed on the maze task. According to the learned drive position, the activity of other individuals in coaction can be quite important in determining their arousal value.

In the mixed conditions, individuals working on the multiplication task should be effectively neutral stimuli for those subjects working on the maze task. That is, the presence of subjects working on the multiplication task should not significantly affect the outcomes of subjects working on the maze task; they are neither competitors nor a source of evaluation. Hence, within 
the high-evaluation condition, groups of homogeneous subjects should be more aroused than groups of mixed subjects. In the homogeneous condition, each subject is being compared to seven others and each subject is potentially a negative stimulus; in the mixed condition, each subject is being compared to only three others (four subjects worked on the maze task). Since the maze task is a complex task and since subjects in the mixed condition should be less aroused than subjects in the homogeneous condition, subjects in the mixed condition should perform better than those in the homogeneous condition. Under lowevaluative conditions, other coactors should be relatively neutral stimuli and not a major source of arousal. Hence, group composition should not affect task performance. The learned drive position also predicts that low-evaluative condition subjects should be less aroused than high-evaluative condition subjects. Since the maze task is a complex task, the performance of low-evaluative subjects should be superior to that of high-evaluative subjects.

\section{METHOD}

\section{Subjects}

Sixty-four psychology students were tested on the maze task and employed as subjects. Subjects arrived at the experiment in same-sexed groups of eight, with an equal number of males and females assigned to each of the four experimental conditions. The scores of four of the sixty-four subjects were deleted from the data analysis due to either failure to follow instructions or malfunctioning equipment.

\section{Task}

The maze task was a "multiple-U" maze machined in Plexiglas (see Hunt \& Hillery, 1973, for details). On this task the starting point is located near the bottom edge of the maze and the goal near the top. The maze has 12 levels, with two alternatives at each level, and is geometrically balanced, requiring as many left as right turns at the decision points in order to correctly trace it with a stylus. A red light on the box which contains and shields the maze comes on when the subject makes an error, and a white light comes on when the subject has reached the goal.

\section{Procedure}

In all conditions, four subjects were seated on each side of the table. In the mixed condition, no two subjects working on the same task were seated either adjacent to or across from each other. Once the subjects were seated, the experimenter read the task instructions. In the homogeneous condition, all subjects were given the maze task instructions, while in the mixed condition, four subjects were told that they would work on the maze task. Maze subjects were told that their task was to find the correct alley connecting each level and to proceed through the maze to the goal located at the top. They were to continue tracing the maze until told to stop. Subjects working on the multiplication task were told that their objective was to multiply the set of numbers as quickly and as accurately as possible.

Subjects in the low-evaluation condition were told not to give the experimenter their name since he was simply having them do this task(s) in groups so that he could obtain a group score. Even though individual scores would not be examined, the subjects were asked to try their best. In the high-evaluation manipulation, subjects were told that several people would be evaluating and comparing their scores. They were told that the experimenter's general hypothesis was that ability in this task(s) might be correlated with other important skills and attributes. Subjects in the mixed condition were told that each task would be examined separately.

Subjects' errors were recorded electrically on counters in an adjacent experimental room. The dependent measure was the number of errors in five trials of maze performance; a trial was completed when the subject reached the goal at the top of the maze.

\section{RESULTS AND DISCUSSION}

The mean number of errors in the four experimental conditions were: high-evaluative mixed $(\bar{X}=21.6)$, high-evaluative homogeneous $(\bar{X}=29.5)$, low-evaluative mixed $(\overline{\mathrm{X}}=26.3)$, and low-evaluative homogeneous $(\overline{\mathrm{X}}=26.1)$. An analysis of variance revealed a significant Group Composition by Evaluation interaction $[\mathrm{F}(1,59)$ $=6.96, p<.05]$. The group composition main effect approached significance $[F(1,59)=3.00, p<.10]$, with mixed condition subjects tending to make more errors than homogeneous condition subjects. Planned comparisons indicated that within the high-evaluation condition the mixed subjects made significantly fewer errors than the homogeneous subjects $(\mathrm{p}<.05)$, while within the low-evaluation condition there was no group composition effect $(F<1)$. These results are consistent with the learned drive prediction. However, counter to the learned drive position, lowevaluation subjects did not perform significantly better than high-evaluation subjects $(F<1)$. Low-evaluation subjects should have performed better than highevaluation subjects since the former were presumably less aroused. Since this was not the case, it is reasonable to assume that another variable may have been operative. It may be necessary to consider both incentive motivation and arousal in investigating the effects of the presence of others. The presence of evaluative others may induce both fear of potential negative outcomes, resulting in increased arousal, and an enhanced motivation to emit those responses that could decrease the probability or intensity of a negative outcome. Individuals with low levels of incentive motivation may not respond with much effort, resulting in a low level of performance; individuals with a high level of incentive motivation may expend a large amount of effort with a resulting high level of performance. High-evaluation subjects were presumably both more motivated and more aroused than low-evaluation subjects. The high level of motivation may have counteracted the effect of the increased arousal, resulting in no overall difference in performance between high- and low-evaluation condition subjects.

\section{REFERENCES}

Cottrell, N. B. Social facilitation. In C. G. McClintock (Ed.), Experimental social psychology. New York: Holt, Rinehart, \& Winston, 1972. 
Hunt, P. J., \& Hillery, J. M. Social facilitation in a coaction setting: An examination of the effects over learning trials. Journal of Experimental Social Psychology, 1973, 9, 563-571.

Paulus, P. B., \& Murdoch, P. Anticipated evaluation and audience presence in the enhancement of dominant responses. Journal of Experimental Social Psychology, $1971,1,280-291$.

Seta, J. J., Paulus, P. B., \& Schkade, J. K. The effects of group size and proximity under competitive and cooperative conditions. Journal of Personality and Social Psychology, 1976, 34, 47-53.

ZAJONC, R. B. Social facilitation. Science, 1965, 149, 269-274.

Zajonc, R. B., \& SAles, S. M. Social facilitation of dominant and subordinate responses. Journal of Experimental Social Psychology, 1966, 2, 160-168.

(Received for publication September 15, 1976.) 